\title{
The relationship between instruction specificity and resistiveness to care during activities of daily living in persons with dementia
}

Jeffrey A. Buchanan, Britta DeJager, Sandra Garcia, Daniel Houlihan, Carolina Sears, Kathleen Fairchild, Adam Sattler Minnesota State University, Mankato, United States

Received: March 15, 2018

DOI: $10.5430 /$ cns.v6n4p45
Accepted: May 1, 2018

Online Published: May 7, 2018

\begin{abstract}
It is common for persons with dementia to display resistant behaviors during activities of daily living (ADLs) and caregiver communication skills can influence the likelihood of these behaviors. Previous research suggests that the type of instructions issued by caregivers is related to rates of compliance during ADLs in persons with dementia. It is unclear, however, if these instruction types affect the likelihood resistiveness to care (RTC) during ADLs. The purpose of the current study was to examine how the use of different types of instructions relate to instances of RTC during ADLs. Results indicated that vague and ambiguous instructions (i.e., beta instructions) were more likely to precede and follow physical aggression compared to instructions that were more specific, clear and feasible (i.e., alpha instructions). Results also indicated that alpha instructions were more likely to precede verbal agitation. These findings have implications for communication training programs for caregivers working with persons with dementia.
\end{abstract}

Key Words: Dementia, Resistiveness to care, Communication

\section{INTRODUCTION}

Dementia (i.e., neurocognitive disorder) is a syndrome that causes cognitive deficits that negatively interfere with independence and daily functioning. ${ }^{[1]}$ Dementia may also involve a range of behavioral disturbances, with resistiveness to care (RTC) during activities of daily living (ADLs) being one of the most challenging to manage. RTC may involve a variety of physically aggressive behaviors (e.g., grabbing, kicking, pushing) and/or verbal agitation (e.g., crying, threatening, screaming) that occur in response to an encounter with a caregiver. ${ }^{[2,3]}$ RTC is estimated to occur in approximately $70 \%$ of individuals with dementia, with an increased likelihood of occurrence in individuals with more severe dementia. ${ }^{[3,4]}$

\subsection{Consequences of RTC}

RTC can have significant consequences for individuals with dementia and their caregivers. For example, RTC may result in the prescription of psychotropic medications, particularly if the behaviors exhibited are physical in nature (e.g., hitting). The Centers for Medicare and Medicaid Services report that approximately $16 \%$ of persons with dementia living in long-term care settings receive antipsychotic medications. ${ }^{[5]}$ Research suggests that the risks associated with these treatments (e.g., increased risk of stroke, cognitive decline, death) outweigh the minimal clinical benefits. ${ }^{[6,7]}$

\footnotetext{
*Correspondence: Jeffrey A. Buchanan; Email: jeffrey.buchanan@mnsu.edu; Address: Minnesota State University, Department of Psychology, 103
} Armstrong Hall, Mankato, MN 56001, United States. 
Psychotropic medications may also further impair remaining abilities such as ambulation and verbal communication, which poses an ethical concern because the treatment causes further loss of functioning in individuals who already are losing function due to the underlying dementia. ${ }^{[7]}$

RTC negatively affects care providers as well. For example, skilled nursing home staff report aggression associated with ADLs as being the most disruptive type of behavioral disturbance and approximately $50 \%$ of nursing assistants report injuries related to physical aggression. ${ }^{[8-10]}$ Furthermore, aggression is associated with caregiver frustration, burnout, and poor job satisfaction. ${ }^{[11,12]}$

\subsection{Communication and RTC}

RTC occurs in the context of ADLs that may require intimate physical contact that can be misperceived as threatening by the person with dementia. Furthermore, language impairments associated with dementia compromise the person's ability to express concerns and/or understand caregiver instructions. Consequently, RTC is conceptualized as defensive behavior because the individual with dementia is attempting to respond to a perceived threat in the environment (e.g., someone taking his or her clothes off) utilizing the remaining physical and verbal capabilities they possess. Therefore, RTC can be conceptualized as a novel means to communicate a need/desire for the ADL context to change because it is frightening or overwhelming. ${ }^{[13]}$ In fact, Talerico et al. found that impaired communication was associated with aggression and the authors suggested that aggression was a primary communicative mode for individuals with severely impaired communication skills. ${ }^{[14]}$ As a result, completing ADLs can be frustrating for caregivers as well as for persons with dementia because both parties find it difficult to express their needs in a manner that can be understood adequately by the other party.

Because persons with dementia are generally unable to acquire new communication skills, research must focus on identifying communication styles of caregivers that may exacerbate communication difficulties that, in turn, can increase the probability of RTC. Indeed, a body of empirical research consistently indicates that caregiver communication style is a common antecedent to RTC. For example, Burgio and colleagues found that staff verbal interactions were more likely than chance to precede agitation in persons with dementia. Furthermore, when agitation occurred, staff tended to respond with continued verbal interactions that could exacerbate agitation in many residents. ${ }^{[15]}$ In the context of ADLs (baths), other researchers have found that that caregiver behaviors such as negative verbal expressions, confrontational communication, invalidation of resident feelings, failure to prepare residents for a task, and disrespectful speech are more likely to occur prior to aggressive behavior. ${ }^{[16,17]}$

\subsection{Elderspeak}

One specific communication style that has been consistently shown to negatively affect patient care is "elderspeak" [18] The hallmark characteristics of elderspeak include using exaggerated intonation, louder volume, higher pitch, less respectful forms of address (e.g., sweetie), and the exclusive "we" (e.g., "Should we get dressed?"). Other common characteristics are debasing nonverbal behaviors (e.g., patting the person's head) and infantilizing language (e.g., "Let's get some food in your tummy"). ${ }^{[19]}$

Caregivers report that elderspeak is intended to make themselves seem friendlier, to make residents feel more comfortable, to improve resident comprehension, and to increase the likelihood that residents will cooperate during caregiving tasks. ${ }^{[20]}$ Research suggests, however, that older adults perceive elderspeak as disrespectful and it may promote greater dependency. ${ }^{[21,22]}$ In addition, collaborative "we" instructions (e.g., "We need go to the bathroom"), one form of elderspeak, do not result in greater compliance in persons with dementia during ADLs. ${ }^{[23]}$ Williams et al. suggest persons with dementia are often aware that people are addressing them in a patronizing fashion and will respond accordingly. For instance, use of elderspeak greatly increases the likelihood of RTC during ADLs. ${ }^{[18]}$ These studies demonstrate that although elderspeak is well intended, it often does not have the expected effects.

\subsection{Verbal instructions}

Completing ADLs with persons with dementia requires caregivers to issue many instructions. Receptive communication deficits associated with dementia, however, can make comprehension and compliance with instructions difficult and can increase the likelihood of RTC. For example, Hart and Wells found that agitation was more likely to follow verbal instructions that were too complex given the comprehension abilities of the participants. ${ }^{[24]}$ Unfortunately, this study did not examine instructions providing in the context of ADLs, but the result do suggest that the kinds of verbal instructions used by caregivers can alter the probability of behaviors similar to RTC.

Fortunately, other studies have examined the relationship between caregiver instructions and RTC (or similar constructs) during ADLs. Roth et al. found that verbal prompts/instructions provided by staff during ADLs were more likely to evoke agitation from residents than expected by chance. ${ }^{[25]}$ After a staff training intervention that involved teaching nursing staff to provide more simple, one-step ver- 
bal instructions, the relationship between verbal instructions and agitation was no longer significant. These findings suggest that prior to communication skills training, staff relied too heavily on issuing verbal instructions that were too complex for persons with dementia to follow.

Christenson and colleagues examined the relationship between the specificity of verbal instructions provided during ADLs and rates of compliance with instructions by persons with dementia. ${ }^{[23]}$ Based on previous research in other populations, the researchers measured two different types of instructions: alpha and beta instructions. ${ }^{[26]}$ Alpha instructions are instructions (i.e., order, suggestion, or question) in which a motoric response is appropriate and reasonable due to it being clear and concise (e.g., "give me your right arm", "stand next to me"). Conversely, beta instructions are instructions in which a person has no opportunity to be compliant due to vagueness, indirectness, or interruption of the instruction (e.g., "stop", "give me that"). The researchers found that $71 \%$ of all alpha instructions resulted in compliance while only $47 \%$ of beta instructions resulted in compliance.

Similarly, Belzil and Vezina examined the relationship of a variety of caregiver physical and verbal behaviors on RTC. ${ }^{\text {[27] }}$ They found that RTC was more likely to occur following negative statements and negative instructions (e.g., instructions guiding the resident on what not to do). This relationship was present not only when the resident was already engaging in RTC, but also when there was an absence of physical aggression on the part of the resident. These results suggest that negative instruction may increase the likelihood of RTC, and may exacerbate RTC when it is already occurring. Another interesting finding was that positive instructions (i.e., instructions guiding the resident on what to do) were also associated with RTC, but only when the instruction took place while RTC was already occurring or when there was an absence of resident physical aggression and the individual had more severe language impairment. These results suggest that positive instructions do not result in termination of RTC that is already occurring and may increase the likelihood of RTC in persons with more severe language impairment.

\subsection{Purpose of the study}

The overarching goal of this study was to gain a better understanding of the complex relationship between instruction specificity and RTC in persons with dementia. Previous research has shown that lack of specificity of instructions may be an antecedent to RTC in persons with dementia. ${ }^{[24]}$ Little research, however, has examined how caregivers respond verbally once RTC occurs. It is important to examine how caregivers respond verbally to RTC once it starts in order to determine if different types of verbal responses are more or

Published by Sciedu Press less likely to perpetuate or terminate RTC. Research with other populations suggests that beta instructions are related to an increase in aggression when issued while potentially violent or stressful situations are occuring. ${ }^{28,29]}$ In the context of dementia care, Belzil and Vezina found that when RTC occurs, most caregiver verbal responses (including negative instructions) were followed by continued RTC. ${ }^{[27]}$ However, Belzil and Vezina did not establish if specific types of caregiver instructions were more or less likely to occur in response to RTC. Therefore, the purpose of this study was to extend the existing literature on the relationship between verbal instructions and RTC to determine how the use of different instruction types by caregivers relate to instances of resident RTC during ADLs.

Consistent with literature in other populations, it was hypothesized that RTC would be more likely to occur following beta instructions by CNAs compared to use of alpha instructions. ${ }^{[28]}$ This hypothesis was based on the ecological model of aging and adaptation that suggests when environmental demands ("press") and the capabilities of the individual are not in balance, negative reactions may occur. ${ }^{[30]}$ In the context of the current study, it is suggested that beta instructions tax the capabilities of persons with dementia such that likelihood of confusion and RTC increases. In addition, it was hypothesized that beta instructions by CNAs in response to RTC would be associated with increased RTC as compared to alpha instructions. This hypothesis was based on the premise that stress caused by the occurrence of RTC would increase the likelihood of using beta instructions.

\section{Methods}

The current study involved analyzing a subset of data from a larger pool of data obtained in a previous study. ${ }^{[23]}$ The research methodology described below in terms of participants, settings, and operational definitions of the measured constructs pertains to this larger study by Christenson and colleagues. An explanation of the specific procedures employed for the current study is included in the "Procedure" section below.

\subsection{Participants}

Two samples of participants were recruited and informed consent was obtained from all participants or their legal guardians (approved by the Institutional Review Board at Minnesota State University, Mankato). The first sample included 11 individuals with dementia (4 males and 7 females). Participants were recruited from long-term care facilities in the Midwestern region of the United States and were nominated by directors at each facility. Directors were asked to select residents who could participate minimally during 
ADLs, lived in the facility for at least three months, and had a diagnosis of dementia. A diagnosis of dementia was confirmed in one of two ways: (1) a chart review to identify the presence of a diagnosis of dementia, and/or (2) participants resided in a memory care unit that required residents to have a dementia diagnosis. All participants were Caucasian.

The second sample of participants included 11 certified nursing assistants (CNAs; 4 males and 7 females). Directors at each facility identified CNAs who worked with each participant regularly (i.e., at least two to three times per week for at least one month). Ten participants identified as Caucasian and one participant identified as Middle Eastern.

\subsection{Setting}

Data collection occurred in three settings. The first setting housed approximately 30 individuals and was a secured special care unit designed for individuals with dementia located in a small Midwestern city. The second setting included a nursing home in a small Midwestern city and housed approximately 90 individuals. The third setting included a nursing home for veterans in a large Midwestern city. This facility included a 3-floor building where individuals with dementia resided.

\subsection{Operational definitions}

Instruction type and RTC were the two general constructs measured in this study. With regard to the type of instructions issued by caregivers, two different categories of instructions were recorded. The first were alpha instructions, which were defined as precise, descriptive orders in which a motoric response was appropriate and feasible. ${ }^{[26]}$ Some examples of alpha instructions observed during ADLs included, "Would you step out of the shower?", "Put your hands up", "Don't hit", "Quit trying to bite me", and "We're going into the bathroom". The second category of caregiver instructions were beta instructions, which were defined as instructions in which the individual is not given an opportunity to demonstrate compliance due to vagueness, interruption, indirectness, or caregiver interference such as carrying out the task for the person. If an instruction that normally would have been coded as a beta instruction (e.g., "move") was accompanied by a clear gesture, the instruction was coded as an alpha instruction. Some examples of beta instructions observed during ADLs included, "Move", "Hold on", "Be nice", "Don't do that", "Quit aggravating me", and "Could you help me?"

Concerning RTC displayed by persons with dementia, two different categories of RTC were recorded in order to separately examine the effects of instruction type on physical and verbal forms of RTC. The first form of RTC that was measured was "physical aggression", which was defined as any instance of physical contact, or any attempt at physical contact towards a caregiver including hitting, slapping, swinging, kicking, grabbing, pushing, scratching, pinching and biting. To account for non-physical forms of RTC, the construct of "verbal agitation" was also measured. Verbal agitation was operationally defined as any instance in which the resident made verbal threats (e.g., "I'm going to bite you", swearing), statements of displeasure or protest (e.g., "stop it", "get me out of here"), or vocal indictors of verbal agitation not involving words such as yelling or groaning.

\subsection{Procedure}

The original dataset from the Christenson et al. study consisted of 27 videotapes of interactions between nursing home residents with cognitive impairment and staff members during various ADLs (e.g., bathing, dressing). ${ }^{[23]}$ To capture these interactions, the researchers placed a video camera in an inconspicuous location (e.g., on a shelf) prior to the start of ADLs and then exited the room. CNAs were then instructed to complete ADLs as they normally would. While ADLs were being completed, the researchers waited outside the room. Upon completion of ADLs, the researchers entered the room to remove the video camera. Researchers transcribed all videotaped observations, which included verbatim accounts of all verbal instructions issued by CNAs, descriptions of physical prompts or gestures that occurred when instructions were issued, and detailed explanations of resident verbal and physical responses to all CNA instructions.

In order to test the current study's hypotheses, a subset of seven tapes from the Christenson et al. study were analyzed. ${ }^{[23]}$ These seven tapes were selected for the current study based on the criteria that they contained physical aggression and/or verbal agitation in at least $10 \%$ of recorded intervals (all videotapes were broken into 5 -second intervals). During each interval, the researchers recorded whether RTC (either physical aggression or verbal agitation) occurred or not. If both physical aggression and verbal agitation occurred, only physical aggression was recorded. If neither target response occurred during a given interval, the interval was coded as a nonoccurrence. After each tape was coded for physical aggression and verbal agitation, each instruction type and the time it occurred during the sessions were recorded. The researchers then recorded if the resident engaged in physical aggression, verbal agitation, or neither response during the interval immediately prior to the instruction, during the interval in which the instruction occurred, or during the interval immediately following the instruction. 


\subsection{Inter-observer agreement}

Two trained research assistants independently coded physical aggression and verbal agitation in $29 \%$ of the seven videos. Using a percent agreement formula [agreements/(agreements + disagreements $) \times 100],{ }^{[31]}$ there was $98 \%$ inter-observer agreement for physical aggression and verbal agitation.

\section{Results}

A total of 1,029 intervals were observed. Of these intervals, $30.8 \%$ of intervals included either physical aggression $(12.2 \%)$ or verbal agitation $(18.5 \%)$. Physical aggression ranged from 0-93 $(\mathrm{M}=18, S D=34.71)$ occurrences in all intervals. Meanwhile, verbal agitation ranged from 9-87 occurrences $(\mathrm{M}=27.29, S D=27.07)$.

Within the 1,029 observed intervals, there were 428 instruc- tions, which included 222 alpha instructions and 206 beta instructions. Four chi-square tests of independence were conducted to determine which instruction types were more likely to precede and follow instances of physical aggression and verbal agitation. Figure 1 displays the percentage of physical aggression before and after staff gave a beta and alpha instruction. A chi-square test of independence demonstrated that beta instructions tend to precede physical aggression $\left[\chi^{2}(1)=5.21, p<.05\right]$, and beta instructions tend to follow physical aggression $\left[\chi^{2}(1)=8.16, p<.05\right]$. Figure 2 displays the percentage of verbal agitation before and after staff instructions. Results indicated that residents were more likely to exhibit verbal agitation after staff members used a beta instruction, $\chi^{2}(1)=5.72, p<.05$; however, staff members are equally likely to use alpha and beta instructions after residents display verbal agitation, $\chi^{2}(1)=1.27, p>.05$.

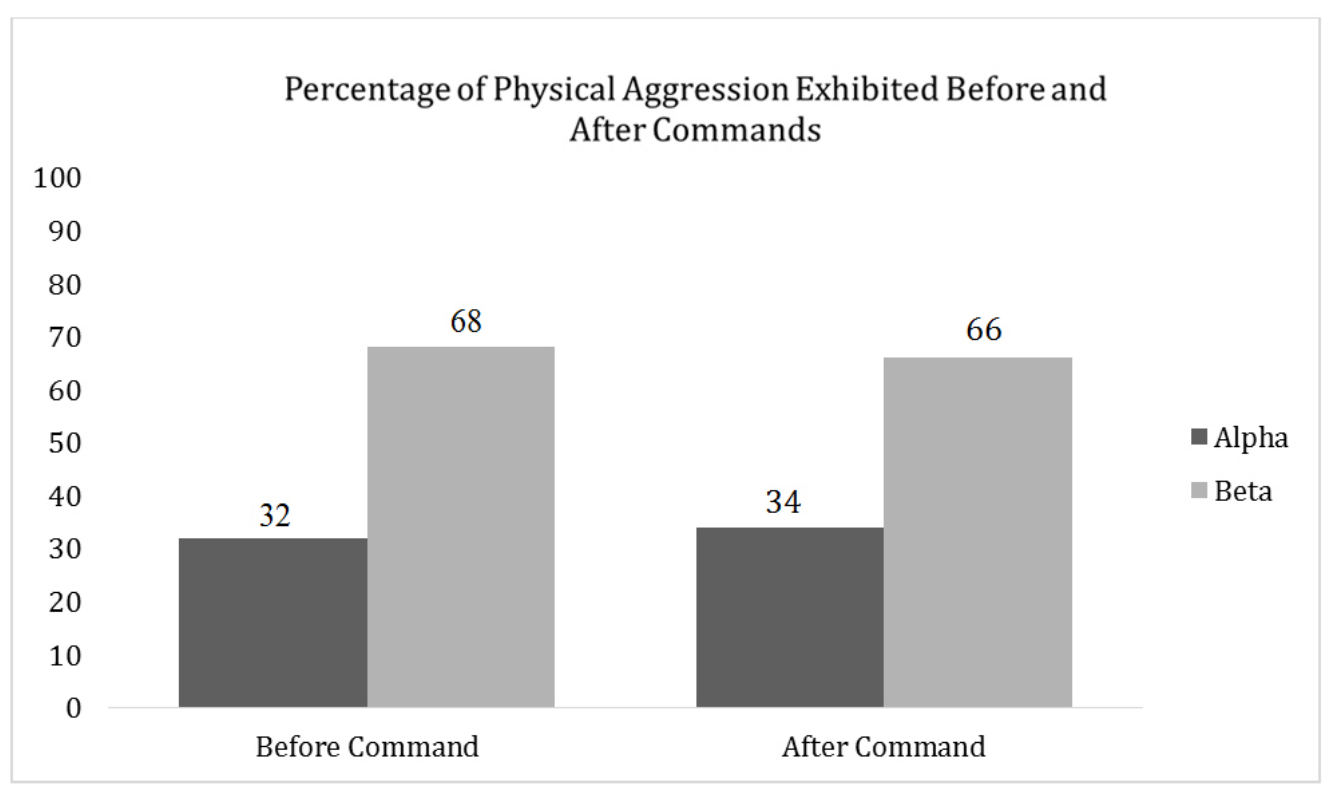

Figure 1. This figure illustrates the percentage of physical aggression that occurs before and after an alpha or beta command is issued

\section{Discussion}

This study adds to the literature concerning how caregiver communication style relates to RTC in persons with dementia. In addition, the study expands the small body of research investigating how caregiver instructions affect older adults with dementia during ADLs. Results indicate an association between beta instructions used by CNAs and physical aggression as well as verbal agitation. The first hypothesis was supported in that beta instructions were more likely to precede physical aggression and verbal agitation compared to alpha instructions. This finding is consistent with existing research showing that more complex instructions and instructions focused on what not to do are more likely to precede RTC during ADLs. ${ }^{[25,27]}$ One possible explanation for this finding is that beta instructions are more difficult for residents with dementia to comprehend due to their vague and indirect nature. Furthermore, ADLs involve intimate physical contact that residents may misinterpret as threatening. The result is that individuals with dementia experience confusion and/or fear, particularly when they have severely impaired expressive and receptive language skills that limit their ability influence the situation in more socially acceptable ways. Consequently, the resident may express these emotions by displaying various forms of RTC.

There was mixed support for the second hypothesis. First, the hypothesis that beta instructions would occur more fre- 
quently following verbal agitation in comparison to alpha instructions was not supported. On the other hand, results did support the hypothesis that beta instructions would occur more frequently following physical aggression. This result is consistent with previous research that found an increased use of beta instructions in police officers during stressful situations, such as when suspects display aggressive behavior. ${ }^{[28]}$ Existing research with persons with dementia also suggests that caregivers commonly respond to agitation and RTC with a variety of verbal responses that perpetuate RTC. ${ }^{[15,25,27]}$ This may occur because of the stressful na- ture of ADLs for both caregivers and persons with dementia. In fact, completing ADLs while a resident displays RTC is amongst the most stressful and disruptive experiences reported by CNAs. ${ }^{[8]}$ The physical and psychological arousal induced by these stressful situations may impair the CNA's higher-order cognitive abilities (e.g., divided attention, problem solving), resulting in continued use of beta instructions despite their ineffectiveness. ${ }^{[32]}$ Likewise, as an encounter escalates, residents may also experience increased arousal, further compromising their ability to comprehend and comply with instructions.

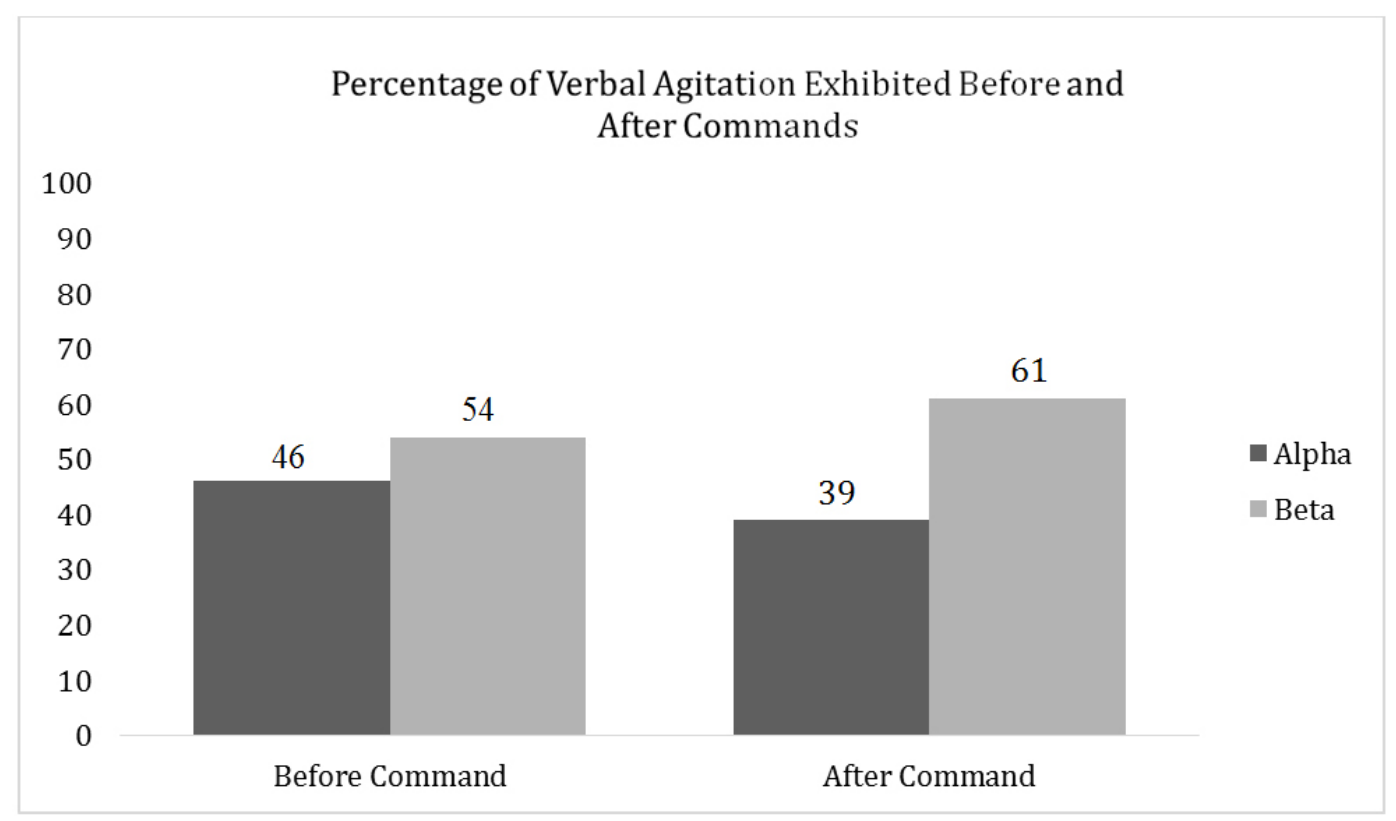

Figure 2. This figure illustrates the percentage of verbal agitation that occurs before and after an alpha or beta command is issued

\subsection{Limitations and future directions}

Several limitations of the study must be acknowledged. First, the sample in this study was small in terms of the number of residents and CNAs as well as the total number of interactions observed. The sample of residents and CNAs was also predominately female and Caucasian. Future research that includes larger and more diverse samples will be necessary to ensure the results of this study generalize to the larger population of caregivers and persons with dementia.

Regarding the sample of older adults, no objective measure of the severity of cognitive impairment was included. Without an objective measure, an analysis of differences in aggression across different levels of cognitive impairment is impossible. Existing research suggests that RTC is more prevalent in residents with more severe cognitive impairment. ${ }^{[3]}$ Nonetheless, future research should include an assessment of how instruction types and resident physical aggression and verbal agitation vary by cognitive impairment severity.

Some limitations are associated with the method of data collection implemented. For example, direct observation methodology inherently involves the risk of reactivity. This risk was minimized by videotaping interactions and placing cameras in inconspicuous places. Although videotapes and transcripts revealed no obvious instances of participants looking at or commenting on the camera, reactivity cannot be ruled out as a potential threat to external validity. In addition, given that data collection involved naturalistic observations of interactions between residents and CNAs, no experimental manipulation of variables occurred. Consequently, there is uncertainty as to whether the association between instruction types and physical aggression and verbal agitation are causal in nature.

Finally, the communication model described earlier is predicated on the notion that CNAs experience stress when chal- 
lenging behaviors occur during ADLs. Although research suggests that these behaviors are often perceived to be quite stressful by CNAs, future research should directly assess how stressful CNAs find specific encounters with residents. ${ }^{[8]}$ It is possible that factors other than the mere presence of RTC (e.g., quality and length of the relationship with the resident), will affect how stressful a CNA perceives an interaction to be. Relatedly, the researchers did not obtain data regarding training in communication skills or the amount of experience CNAs had working with individuals with cognitive impairment. These factors may not only affect how stressful CNAs find specific encounters with residents, but also the quality of their communication skills. Future research should examine if training or years of experience as a CNA results in changes in types of instruction used and if this subsequently affects the frequency of RTC displayed by persons with dementia.

\subsection{Implications}

The results of this study and others suggest a model for understanding the breakdown in communication that can occur during caregiver-resident interactions, particularly during ADLs. The model begins with the CNA providing a vague, indirect statement or request. The vagueness of the request in combination with the impaired communication skills of the resident results in confusion, frustration, and non-compliance on the part of the resident. Rather than clarifying the previous statement or request, CNAs tend to repeat their use of such vague statements, perhaps because of the increasingly stressful nature of the encounter. As caregivers continue to issue beta instructions, the resident eventually engages in behaviors indicative of RTC as a means of communicating their frustration and confusion.

The findings of this study and the model of communication described above have important practice implications. Certified nursing assistants have poor job satisfaction due the stressful nature of the job and inadequate training. ${ }^{[11]} \mathrm{Re}-$ ports have indicated that CNAs are required to complete a minimum of 75 hours of training, but additional training is needed to learn effective behavior management and verbal communication skills. ${ }^{[11,33]}$ Based on current research and the findings of this study, CNAs may rely on ineffective communication styles (e.g., elderspeak, beta instructions) during ADLs that exacerbate an already stressful situation.

Results of the current study have implications for communication-training programs for CNAs. Training programs may include common examples of alpha and beta instructions, videos of how staff issue each type of instruction, and role-playing opportunities. Based on this study and others, this kind of training program may result in greater use of alpha instructions, increased compliance with instructions as well as decreases in RTC during ADLs. ${ }^{[23,25]}$ Possible longer-term outcomes for CNAs include reduced burnout, decreased time needed to complete ADLs, and increased job satisfaction. Furthermore, residents may experience less stress and greater independence during ADLs.

\section{Conclusions}

This study examined whether the types of instructions used by CNAs were related to RTC in persons with dementia during ADLs. Findings revealed that beta instructions were more likely to precede and follow physical aggression. In addition, beta instructions were more likely to precede verbal agitation displayed by residents. Although this study provided valuable insight about the association of instruction use and RTC, future research is needed to replicate these findings and to examine whether staff training has an impact on instruction use as well as RTC exhibited by persons with dementia.

\section{CONFlicts of InTEREST Disclosure}

The authors declare they have no conflicts of interest.

\section{REFERENCES}

[1] Alzheimer's disease facts and figures. Alzheimer's \& Dementia: The Journal of The Alzheimer's Association. April 2016; 12(4): 459-509.

[2] Herman R, Williams K. Elderspeak's influence on resistiveness to care: focus on behavioral events. American Journal of Alzheimer's Disease and Other Dementias. October 2009; 24(5): 417-423. PMid: 19692706. https://doi.org/10.1177/1533317509341949

[3] Volicer L, Bass E, Luther S. Agitation and resistiveness to care are two separate behavioral syndromes of dementia. Journal of The American Medical Directors Association. October 2007; 8(8): 527 532. PMid: 17931577. https://doi.org/10.1016/j.jamda. 20 07.05 .005

Published by Sciedu Press
[4] Keene J, Hope T, Fairburn CG, et al. Natural history of aggressive behaviour in dementia. International Journal of Geriatric Psychiatry 1999; 14: 541-548. https://doi.org/10.1002/(SICI) 1099-1 166 (199907) $14: 7<541:$ : AID-GPS961>3.0.CD;2-P

[5] Centers for Medicare and Medicaid Services. Data show national partnership to improve dementia achieves goals to reduce unnecessary antipsychotic medications in nursing homes. Accessed February 22, 2018. Available from: https://www.cms.gov/Newsroom/MediaReleaseDatabase/ Fact-sheets/2017-Fact-Sheet-items/2017-10-02.html

[6] Sink K, Holden K, Yaffe K. Pharmacological treatment of neuropsychiatric symptoms of dementia: a review of the evidence. JAMA: Journal of The American Medical Association. February 
2005; 293(5): 596-608. PMid: 15687315. https://doi.org/10 $.1001 /$ jama. 293.5.596

[7] Yury C, Fisher J. Meta-analysis of the effectiveness of atypical antipsychotics for the treatment of behavioural problems in persons with dementia. Psychotherapy and Psychosomatics. 2007; 76(4): 213-218. PMid: 17570959. https://doi.org/10.1159/000101499

[8] Cohen-Mansfield J. Agitated behavior in persons with dementia: the relationship between type of behavior, its frequency, and its disruptiveness. Journal of Psychiatric Research. November 2008; 43(1): 64-69. PMid: 18394647. https://doi.org/10.1016/j.jpsych ires.2008.02.003

[9] Hagen B, Sayers D. When caring leaves bruises: the effects of staff education on resident aggression. Journal of Gerontological Nursing. November 1995; 21(11): 7-16. PMid: 7594259. https: //doi.org/10.3928/0098-9134-19951101-04

[10] Squillace M, Remsburg R, Harris-Kojetin L, et al. The national nursing assistant survey: improving the evidence base for policy initiatives to strengthen the certified nursing assistant workforce. The Gerontologist. April 2009; 49(2): 185-197. PMid: 19363014. https://doi.org/10.1093/geront/gnp024

[11] Noelker L, Ejaz F, Menne H, et al. The impact of stress and support on nursing assistant satisfaction with supervision. Journal of Applied Gerontology. August 2006; 25(4): 307-323. https: //doi.org/10.1177/0733464806290935

[12] Pulsford D, Duxbury J. Aggressive behaviour by people with dementia in residential care settings: a review. Journal of Psychiatric and Mental Health Nursing. October 2006; 13(5): 611-618. PMid: 16965482. https://doi.org/10.1111/j.1365-2850.2006.0 0964. $\mathrm{x}$

[13] Fisher J, Drossel C, Ferguson K, et al. Treating persons with dementia in context. Handbook of behavioral and cognitive therapies with older adults. New York, NY, US: Springer Science + Business Media; 2008. 200-218 p. https://doi.org/10.1007/978-0-387-720 07-4_13

[14] Talerico K, Evans L, Strumpf N. Mental health correlates of aggression in nursing home residents with dementia. The Gerontologist. April 2002; 42(2): 169-177. PMid: 11914460. https: //doi.org/10.1093/geront/42.2.169

[15] Burgio L, Butler F, Roth D, et al. Agitation in nursing home residents: the role of gender and social context. International Psychogeriatrics. December 2000; 12(4): 495-511. PMid: 11263716. https://doi.org/10.1017/S104161020000661X

[16] Somboontanont W, Sloane PD, Floyd FJ, et al. Assaultive behavior in alzheimer's disease: identifying immediate antecedents during bathing. Journal of Gerontological Nursing. 2004; 30: 22-29. PMid: 15471060. https://doi.org/10.3928/0098-9134-200 40901-06

[17] Whall A, Colling K, Beck C, et al. Factors associated with aggressive behavior among nursing home residents with dementia. The Gerontologist. December 2008; 48(6): 721-731. PMid: 19139246. https://doi.org/10.1093/geront/48.6.721

[18] Williams K, Herman R, Gajewski B, et al. Elderspeak communication: impact on dementia care. American Journal of Alzheimer's Disease and Other Dementias. February 2009; 24(1): 11-20. PMid: 18591210. https://doi.org/10.1177/1533317508318472

[19] O'Connor B, Rigby H. Perceptions of baby talk, frequency of receiving baby talk, and self-esteem among community and nursing home residents. Psychology and Aging. March 1996; 11(1): 147-154. PMid: 8726380. https://doi.org/10.1037/0882-7974.11.1.147
[20] Grimme T, Buchanan J, Afflerbach S. Understanding elderspeak from the perspective of certified nursing assistants. Journal of Gerontological Nursing. November 2015; 41(11): 42-49. PMid: 26505247. https://doi.org/10.3928/00989134-20151015-05

[21] Baltes M, Wahl H. Patterns of communication in old age: the dependence-support and independence-ignore script. Health Communication. July 1996; 8(3): 217. https ://doi .org/10.1207/s153 27027hc0803_3

[22] Giles H, Fox S, Smith E. Patronizing the elderly: intergenerational evaluations. Research on Language and Social Interaction. 1993; 26(2): 129-149. https://doi.org/10.1207/s15327973rlsi2 $602 \_1$

[23] Christenson A, Buchanan J, Houlihan D, et al. Command use and compliance in staff communication with elderly residents of longterm care facilities. Behavior Therapy. March 2011; 42(1): 4758. PMid: 21292051. https://doi.org/10.1016/j . beth. 201 0.07 .001

[24] Hart BD, Wells DL. The effects of language used by caregivers on agitation in residents with dementia. Clinical Nurse Specialist. 1997; 11(1): 20-23. https://doi.org/10.1097/00002800-1997010 00-00015

[25] Roth D, Stevens A, Burgio L, et al. Timed-event sequential analysis of agitation in nursing home residents during personal care interactions with nursing assistants. The Journals of Gerontology: Series B: Psychological Sciences and Social Sciences. September 2002; 57(5): 461-468. https://doi .org/10.1093/geronb/57.5.P461

[26] Peed S, Roberts M, Forehand R. Evaluation of the effectiveness of a standardized parent training program in altering the interaction of mothers and their noncompliant children. Behavior Modification. July 1977; 1(3): 323-350. https://doi.org/10.1177/014544 557713003

[27] Belzil G, Vézina J. Impact of caregivers' behaviors on resistiveness to care and collaboration in persons with dementia in the context of hygienic care: an interactional perspective. International Psychogeriatrics. November 2015; 27(11): 1861-1873. PMid: 26165352. https://doi.org/10.1017/S104161021500099X

[28] Schwarzkopf EN, Houlihan DD, Kolb K, et al. Instruction types used in police encounters. Law Enforcement Executive Forum. 2008; 8: 99-114.

[29] Vandermay J, Houlihan D, Klein LA, et al. Instruction sequence in police encounters: searching for a linguistic fingerprint. Law Enforcement Executive Forum. 2008; 8(3): 141-151.

[30] Lawton M, Nahemow L. Ecology and the aging process. The Psychology of Adult Development and Aging. Washington, DC, US: American Psychological Association; 1973. 619-674 p. https: //doi.org/10.1037/10044-020

[31] Kelly M. A review of the observational data-collection and reliability procedures reported in the journal of applied behavior analysis. Journal of Applied Behavior Analysis. Spring 1977; 10(1): 97-101. PMid: 16795549. https://doi.org/10.1901/jaba.1977.10-97

[32] Lupien SJ, Maheu F, Tu M, et al. The effects of stress and stress hormones on human cognition: implications for the field of brain and cognition. Brain and Cognition. 2007; 65(3): 209-237. PMid: 17466428. https://doi.org/10.1016/j. bandc. 2007.02 .00 7

[33] Grant LA, Potthoff SJ, Ryden M, et al. Staff ratios, training and assignment in alzheimer's special care units. Journal of Gerontological Nursing. 1998; 24(1): 9-16. PMid: 9510716. https: //doi.org/10.3928/0098-9134-19980101-08 